\title{
Drop Impact on Hot Plates: Contact times, Lift-off and the Lamella Rupture
}

\author{
Sang-Hyeon Lee, ${ }^{\ddagger a}$ Kirsten Harth, ${ }^{\ddagger * b, c}$ Maaike Rump, ${ }^{c}$ Minwoo Kim, ${ }^{a}$ Detlef Lohse, ${ }^{c, d}$ Kamel Fezzaa, ${ }^{e}$ and Jong Ho Je ${ }^{* a}$
}

When a liquid drop impacts on a heated substrate, it can remain deposited, or violently boil in contact, or lift off with or without ever touching the surface. The latter is known as the Leidenfrost effect. The duration and area of the liquid-substrate contact is highly relevant for the heat transfer, as well as other effects such as corrosion. However, most experimental studies rely on side view imaging to determine contact times, and those are often mixed with the time until the drop lifts off from the substrate. Here, we develop and validate a reliable method of contact time determination using high-speed X-ray and Total Internal Reflection measurements. We exemplarily compare contact and lift-off times on flat silicon and sapphire substrates. We show that drops can rebound even without formation of a complete vapor layer, with a wide range of lift-off times. On sapphire, we find a local minimum of lift-off times much shorter than by capillary rebound in the comparatively low-temperature regime of transition boiling / thermal atomization. We elucidate the underlying mechanism related to spontaneous rupture of the lamella and receding of the contact area.

\section{Introduction}

The impact of liquid drops on a heated substrate can be easily observed in a hot pan during cooking: If the pan is hot enough, droplets will levitate on a layer of their own vapor, move seemingly frictionless and evaporate only very slowly. Upon impact from some initial height, they will even rebound and jump several times. At lower surface temperatures, the drops touch the substrate, and display violent boiling behavior with numerous bubbles and spray. Such droplets will evaporate in much shorter time than the levitating ones. The original description of these phenomena goes back to Boerhave ${ }^{1}$ and Leidenfrost ${ }^{2[3}$, see also the review by D. Quere ${ }^{4}$. They have since been observed in many everyday and engineering situations, e.g., in spray cooling of hot metals or electronics $\frac{5}{5}$, in spray combustion $\frac{8}{8}$ or in the cooling of fuel rods in nuclear power plants in case of an accident 9 . In such situations, controlling their deposition and the heat transfer between the solid and the droplets is crucial, because improper droplet behavior can lead to material failure or to increased corrosion. Besides material parameters, the heat transfer between the substrate and the drop is determined mainly by the duration and extent of the contact between the liquid and the solid surface.

Drops which are gently deposited on a hot smooth surface show an immediate transition from contact boiling to film boiling at the static Leidenfrost point $T_{L, s}$. This transition temperature is influenced by parameters of the liquid and of the solid, e.g., accounting for the rate of evaporation and for cooling effects. The loss of contact between the substrate and the liquid at $T_{L, s}$ is accompa-

${ }^{a}$ X-ray Imaging Center, Department of Materials Science and Engineering, Pohang University of Science and Technology, 77 Chengam-Ro, Nam-Gu, Pohang, 37673, Repbulic of Korea.E-mail: jhje@postech.ac.kr

${ }^{b}$ Institute of Physics, Otto von Guericke University Magdeburg, Universitätsplatz 2, 39106 Magdeburg, Germany. E-mail: kirsten.harth@ovgu.de

${ }^{c}$ Physics of Fluids, Max Planck Center and University of Twente, Drienerlolaan 5, 7500NB Enschede, The Netherlands.

d Max Planck Institute for Dynamics and Self-Organization, Am Faßberg 17, 37077 Göttingen, Germany.

e X-ray Science Division, Advanced Photon Source, Argonne National Laboratory, 9700 South Cass Avenue Argonne, Illinois 60439, USA.

$\ddagger$ These authors contributed equally to this work and share first authorship of this article. nied by an immediate increase in the droplet's lifetime, and vice versa a decrease in the heat transfer rate away from the substrate to the droplet 10 . The shape of such static Leidenfrost drops is essentially determined by a balance of capillary and gravitational forces. Typically, an upward vapor bulge forms near the symmetry axis at the drop's underside ${ }^{11-13}$. When the drop exceeds a critical size, this shape becomes unstable leading to lamella rupture and the upward ejection of vapor bubbles 10 . Even solid objects can display the Leidenfrost effect when approaching a hot plate slowly enough 14 15.

The situation gets more complicated when a drop impacts onto the hot surface with some significant velocity. To distinguish it from the usually lower static Leidenfrost temperature of gently deposited drops, this was termed the dynamic Leidenfrost effect 16 . Here, the transition boiling regime arises between full contact and complete levitation. In this case, the radially outward region of the lamella loses contact with the substrate and levitates while the central region retains contact 17 . When the substrate cooling through the interaction with the drop is strong, all regimes may be observed during a single impact event 18 .

Another complication arising from the impact situation is the dynamics of the vapor layer. First, a thin layer of the ambient gas is entrained between any impacting drop and a smooth surface at ambient pressure ${ }^{1920}$. This allows a contact-less rebound even on smooth non-heated substrates for very low Weber numbers, We $=\rho U_{0}^{2} D_{D} / \gamma<5^{20}$ with $U_{0}$ the impact velocity, $D_{D}$ the drop diameter, $\rho$ the density and $\gamma$ the surface tension of the liquid. The drop's bottom deforms to a dimple upon approach, leading to initial contact along a ring 21 23. On a hot surface, vapor is produced additionally, so that the velocity dependence of the transition temperature to film boiling, the dynamic Leidenfrost point $T_{L, d}$, is a priori not clear 16 . It cannot be determined from side view imaging, frequently applied in the literature. High-Speed Frustrated Total Internal Reflection (TIR) imaging allowed the determination of the dynamic Leidenfrost transition, and yields increasing $T_{L, d}$ with increasing initial substrate temperature. Measurements were based on the initial approach of the drop 17|18. The same technique can also elucidate the time-dependent contact morphology, e.g., revealing different kinds of fingering structures 24,25 
and oscillatory wetting states ${ }^{2526}$, or solidification in vicinity of

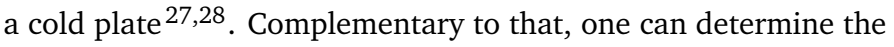
height profile of the liquid/vapor contact line projected onto a 2D plane from Ultrafast X-ray phase contrast imaging 29.32. From this, the existence of contact is not obvious due to limited spatial resolution. Our new method employing X-ray refraction overcomes this problem.

The time scale that a droplet resides in the vicinity of a hot plate (the lift-off time) depends on numerous factors and it ranges from a few milliseconds to seconds depending on the boiling scenarios 33 . The literature is not concise about those scenarios, usually classified on the basis of spray formation, rebound and decomposition detected in conventional side and top view imaging is employed, see e.g. Refs $\frac{16 / 35+38}{3}$. Drop rebound was observed even in contact situations 35 39. The formation of spray drops and other fragments induced by the rupture of vapor bubbles through the lamella is summarized under the term "secondary atomization" 39140 . Spray formation due to bubbles bursting through the lamella is expected at the time when the thickness of the thermal boundary layer reaches the local lamella thickness ${ }^{39}$. Expanding macroscopic holes can induce breakup into comparatively large fragments 34339141 .

Here, hole formation can be thermally induced by lamella rupture in the contact region and independent of $\mathrm{We}^{39}$. The lamella rupture in particular does not require the introduction of topographical defects on the substrate as in Refs. ${ }^{41 / 42}$. For general aspects related to rupture and fragmentation of thin fluid films see the review by Villermaux ${ }^{43}$ and, e.g., Refs. 44 49. The rebound of spread-out droplets before retraction (pancake rebound) reduces the contact time with superhydrophobic substrates ${ }^{42150}$. Due to their large solid-liquid contact angles and low contact-angle hysteresis, drop spreading and receding behavior is in some respect expected to be similar to Leidenfrost drops.

Contact and lift-off times of drops impacting on hot substrates have been only insufficiently discriminated in the literature due to the lack of proper measurement techniques. For water, contact times measured from side and bottom views ${ }^{34139}$ were reported to be roughly independent of the substrate temperature and We in the range of $T_{S}=[210 \ldots 290]^{\circ} \mathrm{C}$ (regime of secondary atomization), but scaling as $\mathrm{Re}_{0}^{-4 / 5}$ with the Reynolds number $\operatorname{Re}_{0}=\rho_{0} U_{0} D_{D} / \eta_{0}$ at ambient temperature, where $\eta_{0}$ is the dynamic viscosity. Liang et al. $\frac{37}{}$ report residence times (from side views) of ethanol and butanol drops independent of the surface temperature in the range of $T_{s}=[200 \ldots 400]^{\circ} \mathrm{C}$ for We $<100$ (without details of the boiling regime), which are in good agreement with the correlations from Biance et al. $\frac{51}{5}$, Chen et al. $\frac{52}{5}$, and their own suggested empirical relation, which all roughly follow a scaling $\propto \mathrm{We}^{0.5}$. The residence times, boiling and atomization regimes are also affected by the wettability of the substrate ${ }^{38}$, with atomization preferentially present on low-contact-angle substrates. However, in particular substrates with very high contact angles are usually not flat, with the topography introducing additional complexity for, e.g., heat transfer, vapour flow and bubble dynamics.

Our interest in this study is a more reliable determination of the contact time and the lift-off or rebound characteristics of im- pacting drops on well heat-conducting hot smooth substrates. TIR can only be employed on transparent substrates, i.e., metal surfaces are excluded. X-ray projection imaging using the phase and absorption contrast of synchrotron X-rays in interaction with the drop can visualize the vapor structures within the liquid droplets independent of the substrate's transparency. Our new approach using X-ray refraction data, validated against TIR measurements below, provides access to contact times of impacting drops. Moreover, we relate details of the contact morphology to conventional side and top view videos. We distinguish four impact regimes by the lift-off, contact and spray formation characteristics, and relate these transitions to the droplet's dynamics and to the lift-off times.

We first explain the experimental setup (Sec. 2). Then, in Sec. 3, we provide a classification of contact / impact regimes, introduce and validate our new method to measure the contact times from X-ray refraction and last determine contact and liftoff times as well as processes on smooth silicon and sapphire. Sec. 4 addresses the surprising appearance of a local minimum of contact times in the transition boiling regime, whose physical background we subsequently explain. This paper ends with conclusions and an outlook (Sec. 5).

\section{Experimental Methods}

We study ethanol drops impacting on bulk optically smooth silicon disks (from Thor Labs in X-ray, thickness $2 \mathrm{~mm}$ ) and sapphire substrates (disks of 2 or $3 \mathrm{~mm}$ thickness obtained from Edmund Optics and Crystan Ltd. in the X-ray experiments, sapphire prism from Crystan Ltd. for TIR). The setups together with example images are shown in Fig. 1 The motivation for using these substrates is avoiding substrate cooling during the interaction with the drop as much as possible, given for the silicon window, and the need of transparency for the direct observation of the contact dynamics using TIR.

\subsection{General Aspects}

We study the impact of ethanol droplets of diameter $2 \mathrm{~mm} \leq$ $D_{D} \leq 2.1 \mathrm{~mm}$ from heights between 4 and $10 \mathrm{~cm}$, corresponding to low impact velocities between $\approx 0.88 \mathrm{~m} / \mathrm{s}$ and $1.4 \mathrm{~m} / \mathrm{s}$. Droplets were produced by pinch-off due to a slow volume increase using a syringe pump. If not noted differently, we used steel needles of standard gauge $21 \mathrm{G}$ and 22G (inner diameter 0.5 and $0.6 \mathrm{~mm}$, outer diameters 0.8 and $0.9 \mathrm{~mm}$, respectively). The temperature of the droplet was not controlled. However, in particular at increased temperatures we discarded a few drops prior to the actual experiment, in order to avoid assembled liquid contamination due to evaporation. The Reynolds and Weber numbers for a $D_{D}=2 \mathrm{~mm}$ diameter drop impacting at $U_{0}=1 \mathrm{~m} / \mathrm{s}$ (the standard case for most experiments reported here) are $\operatorname{Re}=\rho_{0} U_{0} D_{D} / \eta_{0} \approx 1380$ and $\mathrm{We}=\rho_{0} U_{0}^{2} D_{D} / \gamma_{0}=$ 71.2 , respectively, using the values of the dynamic viscosity $\eta_{0}=1.14 \cdot 10^{-3}$ Pas ${ }^{53}$, density $\rho_{0}=786.9 \mathrm{kgm}^{-354}$ and surfac tension $\gamma_{0}=22.1 \cdot 10^{-3} \mathrm{Nm}^{-155}$ of ethanol at room temperature $\left(22^{\circ} \mathrm{C}\right)$. These parameters change to $\eta_{b}=0.42 \cdot 10^{-3} \mathrm{Pas} \mathrm{s}^{53}$, 
(a)

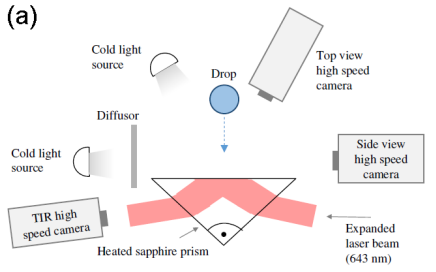

(b)
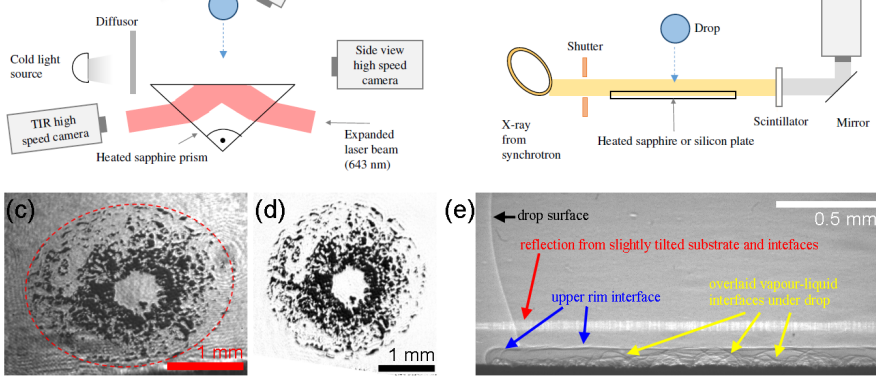

(e)

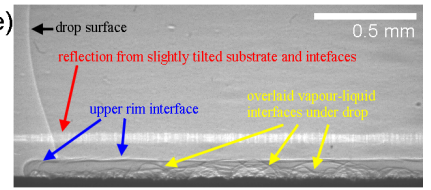

Fig. 1 Schematic of the experimental setups for (a) high-speed TIR combined with synchronized side and top view imaging and (b) ultrafast phase contrast X-ray imaging. Example images show (c) an original frame of the TIR data, (d) the corresponding processed TIR image (see text) and (e) an X-ray image with annotations regarding the visible interfaces and reflections. The contact morphology and local thickness of the vapour layer is highly dynamic and inhomogeneous, as seen by the distribution of the contacting (black) spots in (c,d) and the profile in (e). The data show a $R=1 \mathrm{~mm}$ ethanol drop impacting at $U \approx 1 \mathrm{~m} / \mathrm{s}$ on a sapphire substrate heated to $T_{s}=160^{\circ} \mathrm{C}$ at $t=0.48 \mathrm{~ms}(\mathrm{c}, \mathrm{d})$ and $t=0.31 \mathrm{~ms}$ (e) after impact. The dashed line in (c) indicates the rotated, elliptical appearance of the drop's footprint due to the optical method.

$\rho_{b}=727.9 \mathrm{kgm}^{-354}$ and $\gamma_{b}=17.1 \cdot 10^{-3} \mathrm{Nm}^{-155}$ at the boiling point of ethanol, $T_{b}=78.3^{\circ} \mathrm{C}$, thus $\mathrm{Re}_{b}=3466$ and $\mathrm{We}_{b}=85.2$. The latent heat of vaporization and the isobaric specific heat capacity are $L=841 \mathrm{~kJ} \mathrm{~kg}^{-1[56}$ and $c_{p}=3.182 \mathrm{~kJ} \mathrm{~kg}^{-1} \mathrm{~K}^{-1[57}$, respectively. We use the values at room temperature in the following, as the actual temperature due to heating of the drop has not been measured while it was hanging from the needle.

Both substrates are partially wetting for ethanol at room temperature. For ethanol on untreated silicon, one can extrapolate to a static contact angle of $14.5 \pm 3^{\circ}$ from Ref. $\frac{58}{5}$. We expect a similar contact behavior for ethanol on sapphire. After receding, a sessile drop forms a spherical cap of contact angle $22 \pm 2^{\circ}$. Note that dynamic and static contact angles in non-isothermal situations are usually strongly affected by thermal effects.

The droplets directly impact onto the silicon or sapphire substrates. Given temperatures refer to the calibrated initial surface temperature $T_{s}$. The thickness of the thermal boundary layer in the substrate can be estimated from the scaling $d_{\mathrm{th}} \propto \sqrt{\alpha t} \frac{18}{\text {, }}$ where $t$ is the duration of substrate-liquid contact. The silicon window has a thermal diffusivity of $\alpha_{S i} \approx 0.4 \cdot 10^{-4} \mathrm{~m}^{2} \mathrm{~s}^{-159}$ at $180^{\circ} \mathrm{C}$. For the sapphire substrates, $k_{s, S a}=36 \mathrm{Wm}^{-1} \mathrm{~K}^{-1}$ at $180^{\circ} \mathrm{C}^{60}, \rho_{S a}=3960 \mathrm{~kg} \mathrm{~m}^{-3}$ at $180^{\circ} \mathrm{C}$, $C_{p, S a}=101.7 \mathrm{~J} \mathrm{~mol}^{-1} \mathrm{~K}^{-1}$ at $180^{\circ} \mathrm{C}^{62}, m_{m o l, S a}=101.96 \mathrm{~g} \mathrm{~mol}^{-1}$, leading to a thermal diffusivity $\alpha_{S a}=k_{s, S a} m_{m o l, S a} /\left(\rho_{S a} C_{p, S a}\right) \approx 10^{-5} \mathrm{~m}^{2} \mathrm{~s}^{-1}$. The resulting thickness of the thermal boundary layer, after our time scale of drop spreading of $3.5 \mathrm{~ms}$ at the given $\mathrm{Re}$ and $\mathrm{We}$, is $\approx 22 \mu \mathrm{m}$ in

* The density was estimated using the density value at room temperature, $3970 \mathrm{~kg} \mathrm{~m}^{-361}$, and assuming weighted linear thermal expansion so that $V(T)=$ $V\left(20^{\circ} \mathrm{C}\right)\left[1+\beta_{\|}\left(T-20^{\circ} \mathrm{C}\right)\right]\left[1+\beta_{\perp}\left(T-20^{\circ} \mathrm{C}\right)\right]^{2}$ with the linear coefficients of expansion $\beta_{\|}=5.6 \cdot 10^{-6}{ }^{\circ} \mathrm{C}$ and $\beta_{\perp}=5.0 \cdot 10^{-6}{ }^{\circ} \mathrm{C} 61$ silicon and $\approx 10 \mu \mathrm{m}$ in sapphire. Thus, we assume that substrate cooling is negligible in our experiment, similar to Ref.18.

In the X-ray experiments, $T_{S}$ was varied between $T_{s}=100^{\circ} \mathrm{C}$ and $600^{\circ} \mathrm{C}$ with $1 \%$ accuracy to cover all boiling regimes from contact to film boiling using an electronic heater (SU-200-IH, Maivac). It was directly connected to a thermocouple (UNI-T UT 325) placed in the copper block, and $T_{S}$ was calibrated separately on the substrate's surface using a PT100 probe. The substrates were directly placed on the copper block of the heater. The setup is as described in Refs. 3132 .

The TIR experiments were performed separately. In this case the substrate was a right-angle sapphire prism displaying a top surface of $25 \times 35.35 \mathrm{~mm}^{2}$ placed in a custom-made heated aluminum block. The surface temperature could be controlled between room temperature and $358^{\circ} \mathrm{C}$ with an accuracy of $\approx 1.5 \mathrm{~K}$ using a proportional-integral-derivative controlled electrical heating system. The surface temperature was directly measured and calibrated against the heater set-point using a PT100 temperature sensor flat attached to the surface. The general setup and TIR method have been previously described in Ref. 63 .

\subsection{Imaging Techniques}

Ultrafast synchrotron X-ray imaging were conducted at the 32ID undulator beamline of the Advanced Photon Source, Argonne National Laboratory, to achieve high temporal and spatial resolutions, with a 472 ns exposure time for each frame. Using the setup shown in Fig. 1(b), we are capable of directly visualizing the interfaces between gas and liquid even within the liquid drop at a maximum time resolution of up to $\approx 271,000$ frames per second (fps). The imaging system consists of a fast scintillator and a mirror coupled to a high-speed camera (Photron Fastcam SA-Z) via a microscope objective lens $(10 \mathrm{x}$ with $\mathrm{NA}=0.21)$, resulting in a spatial resolution of $2 \mu \mathrm{m} /$ pixel. Typical frame rates used in the present measurements are between 20,889 and 90,000 fps. As the setup had to be remotely controlled, a laser triggering system was used to sense the falling drop and to trigger the camera and the $\mathrm{X}$-ray beamline shutter. The method has been successfully applied previously to drops impacting on non-heated solid substrates and onto liquid layers $\frac{64}{66}$ as well as heated substrates $29 / 31 / 32$. Heating of the scintillator due to the intense radiation limits the total measurement duration to $\approx 40 \mathrm{~ms}$.

The strength of edge-enhanced x-ray phase contrast imaging is the possibility to visualize both the drop liquid and the vapor phase as demonstrated in Fig. 1(e), and the ability to detect in particular even small distortions of liquid-gas interfaces irrespective of substrate transparency. Measurements were performed in transmission, so that all interfaces in the direction of the beam are overlaid on a single image. An example of an ethanol drop impacting onto a sapphire disk heated to $T_{s}=160^{\circ} \mathrm{C}$ (surface slightly tilted toward the incoming X-ray beam, tilt angle $<0.1^{\circ}$ ) is shown in Fig. 1 (e), and the appearance of the gas-liquid interfaces and the substrate is clarified. For the later analysis, the substrate was positioned horizontally, by adjusting the tilt so that the reflection barely disappears at the substrate. Minimal tilt corresponds to the minimal height at which the black 
substrate region appears in absence of the drop. The refraction in the substrate region remains and will be analyzed. Due to the limit of the spatial resolution and the typically immense number of bubbles forming directly at the substrate in the transition boiling regime, the existence of contact is not obvious. We developed a novel approach using the structure of the X-ray refraction at the gas-liquid interfaces underneath the drop (see below). Note that the hard X-ray irradiation causes negligible heating and vaporization of the liquid or change of the properties of liquids for the very short exposures used here $(<300 \mu \mathrm{s}) 67$.

High-speed Frustrated Total Internal Reflection (TIR) imaging relies on the fact that light is totally internally reflected at the interface between an optically dense and an optically dilute medium when exceeding a critical angle of incidence. This allows a reliable detection of wetted regions on the substrate, apart from temperatures close to the dynamic Leidenfrost point when the contact features approach the limit of experimental spatial and temporal resolution. With some limitations, also accurate quantitative height data in the range of the evanescent wave can be retrieved 63 . Our TIR setup was combined with synchronized side view imaging, and for part of the experiments also tilted top view imaging, as sketched in Fig. 1(a).

For the TIR sketched in Fig. 11(a), we introduce a s-polarized expanded continuous laser beam, $\lambda=643 \mathrm{~nm}$, of roughly 2 $\mathrm{cm}$ spot diameter into our sapphire prism (refractive index $n=1.76^{61}$ ) at an angle such that it is totally internally reflected at the gas-sapphire interface, while it is transmitted into the ethanol drop in contact. The actual angle is not important for the current measurement, but can be calculated from the distortion of the recorded TIR images ${ }^{63}$. Under these conditions, all spots wetted by the drop appear dark in the images. As seen in Fig. 1(c), the original recorded images distort the circular drop footprint to an ellipse, whose axis is additionally rotated to the frame borders as we used a second mirror to guide the reflected beam to the camera lens. All images shown below are normalized by their background and undistorted, exemplarily shown in Fig. 1(d). TIR recordings presented here were recorded at frame rates between 30,000 and $80,000 \mathrm{fps}$ at a spatial resolution in the raw images of typically $7.45 \mu \mathrm{m} / \mathrm{pixel}$, and few low temperature data sets at $12.3 \mu \mathrm{m} /$ pixel.

In side view, we employed a cold light source and a diffusor to provide backlight. For the tilted top view, a cold light source was placed at a mirrored opposing position to the camera lens, such that the light reflected on the prism's surface provides a bright background. These recordings were employed for extraction of the drop size, impact velocity, the lift-off or rebound times (side) and to observe the hole formation morphology (tilt view). The spatial and temporal resolution varies between data sets, depending on the process of interest, we employed frame rates between 4,000 and 30,000 fps, most side view recordings possess spatial resolutions between $12 \mu \mathrm{m} /$ pixel and $20 \mu \mathrm{m} /$ pixel.

\section{Contact Regimes, Contact and Lift-off Time}

First, we categorize our impacting drops into four main regimes observed at an impact velocity of $U \approx 1 \mathrm{~m} / \mathrm{s}$, based on the contact and the lift-off / rebound characteristics seen in the vapor layer dynamics. In particular, the distribution of contact locations on the substrate is by far not spatially homogeneous, see Fig. 2. We define the dynamic Leidenfrost point as the lowest temperature where no contact between the drop and the substrate is observed during the entire spreading and receding phase, which is identified by combination of TIR and X-ray, details will be discussed

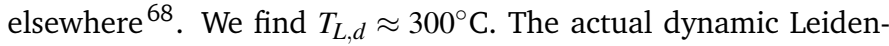
frost point may be slightly $\left(1020^{\circ} \mathrm{C}\right)$ lower under cleanroom conditions. Nevertheless, this is substantially higher than the value of $200^{\circ} \mathrm{C}$ to $220^{\circ} \mathrm{C}$ based on the initial contact alone reported by Shirota et al. $\frac{17}{}$, and similarly by Khavari and $\operatorname{Tran}^{26}$. In repeated experiments, contact was occasionally also observed at temperatures up to $340^{\circ} \mathrm{C}$, with decreasing probability. A transitional boiling regime is found in a broad range of temperatures between the contact and Leidenfrost boiling regimes 17,18 . Then, the maximal radius of the wetted area in TIR is equal to or smaller than the spreading radius observed in top or side view, respectively. We observe a lower limiting temperature for transition boiling of $(161 \pm 2)^{\circ} \mathrm{C}$. Oscillatory wetting ${ }^{25 \mid 26}$, where radially inward traveling wetting fronts characterize the TIR recordings, is already observed at slightly lower surface temperatures of $156^{\circ} \mathrm{C}$. As we are interested in the contact and lift-off times, we will adopt a different classification here.

Second, we determine the contact and lift-off or rebound times of ethanol drops on sapphire and silicon substrates. Atomization gives rise to a local minimum of the lift-off time on sapphire. We qualitatively explain its relation to the receding contact in the transition boiling regime and the localized rupture of the lamella in Sec. 4

\subsection{Impact Scenarios}

The four distinguished impact and contact regimes with their appearance in optical and X-ray data are exemplarily shown in Figs. 2 and 3 and schematically drawn in Fig. 4 . The scenarios are similar on sapphire and silicon substrates, as well as for the entire investigated range of impact velocities.

Regime I, Figs. 2(a), 3(a) and 4(a), corresponds to the contact boiling regime, where drops impact onto a substrate of $T_{S}$ sufficiently high above the boiling point, but at comparatively low temperatures. They spread on the substrate and start violently boiling. Numerous vapor bubbles nucleate, grow and sometimes merge, and subsequently burst through the drop's surface or sideways near the contact line of the drop on the substrate, until the drop is completely decomposed into smaller spray drops and the main drop evaporated on the substrate. The contact times are of the order of $0.5 \mathrm{~s}$ at $T_{s}=160^{\circ} \mathrm{C}$ in our experiment. The numerous moving contact lines and often millimeter-sized bubbles are easily identified in the optical and X-ray data.

In Regimes II-IV, the droplets lose contact with the substrate 


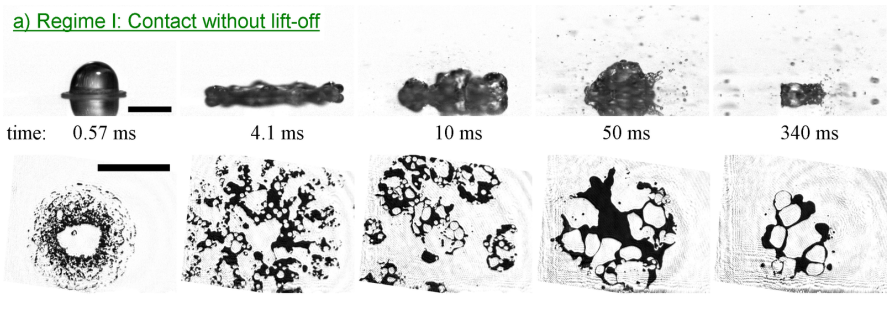

b) Regime II: Contact until lift-off, thermal atomization
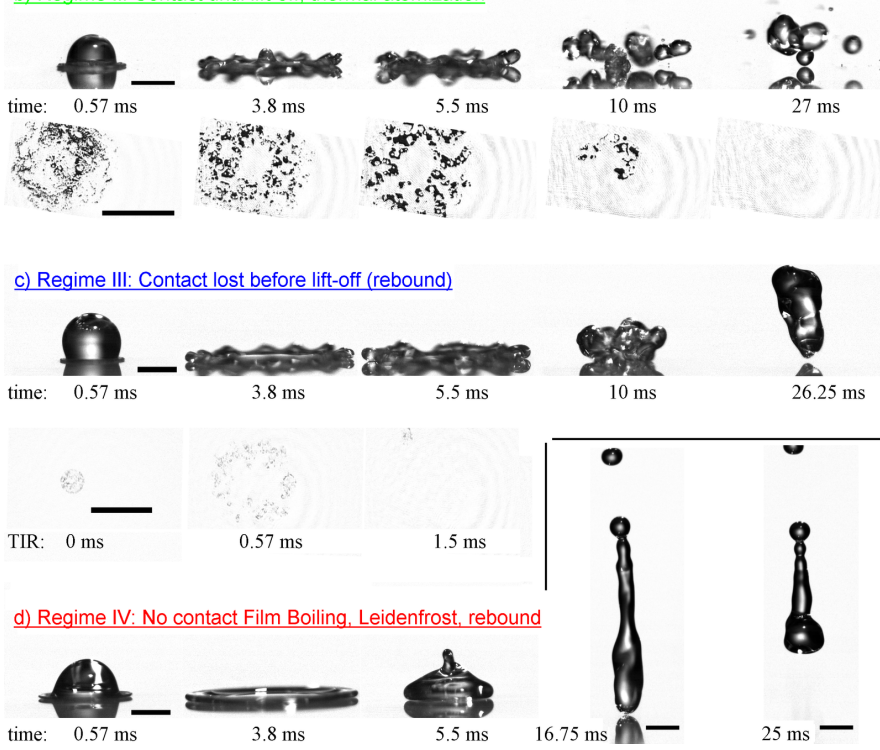

Fig. 2 Contact and lift-off regimes in optical measurements, see corresponding Fig. 3 for X-ray: Ethanol, drop diameter $2 \mathrm{~mm}$, impact velocity $1 \mathrm{~m} / \mathrm{s}, W e=71.6$, initial sapphire substrate temperatures $T_{s}$ (a) $160^{\circ} \mathrm{C}$, (b) $168^{\circ} \mathrm{C}$, (c) $204^{\circ} \mathrm{C}$ and (d) $323^{\circ} \mathrm{C}$. (a) Regime I - drops stick to the plate, accompanied with strong spray formation until complete evaporation. (b) Regime II - drops stick to the plate until the retraction releases the contact so that lift-off occurs (compare TIR and side view), usually accompanied by (upward and sideways) spray and sometimes drop fragmentation; (c) Regime III - contact vanishes (no TIR signal after $1.6 \mathrm{~ms}$ ) long before the drop rebounds, (d) Regime IV - Leidenfrost drops, which never touch the substrate. Time is given after the first frame seen in TIR (a-c), and from contact in side view in (d) as there is no contact seen in TIR. See text for detailed regime description. Scale bars are $2 \mathrm{~mm}$ in each sub-figure. Note the slightly different scale between the time ranges in (d) $[0.57 \ldots 5.5] \mathrm{ms}$ and (d) $[10,26.25] \mathrm{ms}$.

prior to complete evaporation (identified by TIR and X-ray data). This can occur by a classical rebound, where the drops jump to a significant height. In particular at lower temperatures, the drops (or their fragments) rise from the substrate at small velocity to a clearly visible but small height (much less than $R_{0}$ ), see Fig. 4 (b). As we would like to distinguish this from the classical rebound, we rather generalize the term to 'lift-off', including any of the two situations.

The lift-off and contact times, $t_{l}$ and $t_{c}$, are often not cleanly distinguished in the literature, as this is impossible from simple side and top view data. The literature often presents $t_{c}$ as the visible loss of contact with the substrate in side view or the lack of visible structures on the lamella from top view 239 . One the one hand, this causes an overestimation of $t_{c}$ in Regimes II and III. On the other hand, the dynamic Leidenfrost transition is underestimated

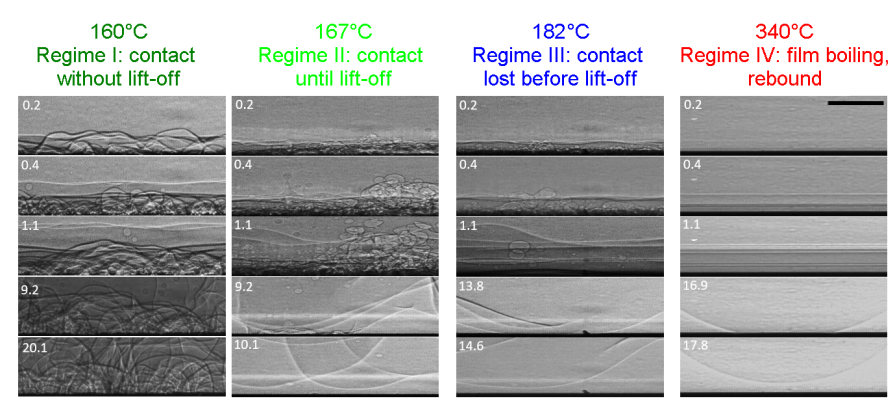

Fig. 3 Contact and lift-off regimes of drops impacting on a smooth hot plate detected by side view X-ray imaging, description as in Fig. 2 Numbers give the time after impact in ms. Note the persisting contact until $t=9.2 \mathrm{~ms}$ in Regime II, compared to the early loss of contact in Regime III. The length of the scale bar is $0.2 \mathrm{~mm}$ and applies to all images. The Weber number is 71.2 , drop diameter $2 \mathrm{~mm}$.
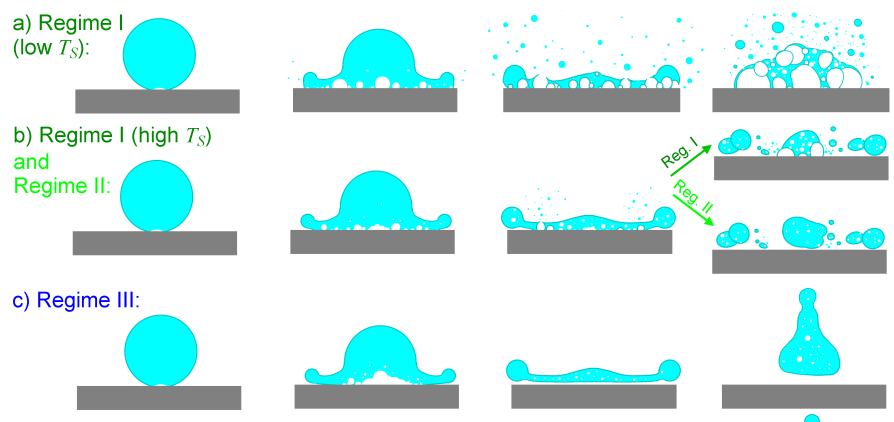

d) Regime IV: Leidenfrost
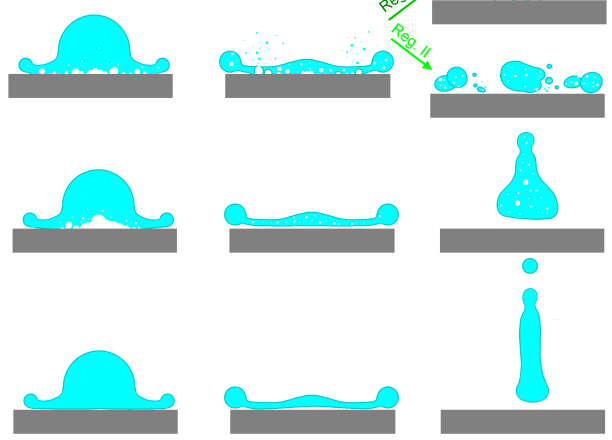

Fig. 4 Sketch of the drop behaviour in the 4 impact regimes, Figs. 2 and 3 All cases are similar at impact (first column), vapour formation proceeds faster with increasing $T_{S},(a-d)$, and column 4 shows the impact outcome after drop receding.

due to very short and sparse contacts at high temperatures 68 .

We extract the lift-off time, $t_{l}$, from the side views in phase contrast X-ray and TIR data. We also determine the actual contact time, $t_{c}$, which is often much smaller than $t_{l}$ (see below). Note that structures on the spread out lamella in optical top view images may persist for a while after contact was already lost, and are thus not an indication of persisting contact.

In Regime II, Figs. 2(b), 3(b) and 4(b),part of the drop continuously remains in contact with the substrate until lift-off. At a temperature of $T_{s}>165^{\circ} \mathrm{C}$ (for ethanol on sapphire, $D_{D}=2 \mathrm{~mm}$, $U_{0}=1 \mathrm{~m} / \mathrm{s}$ ), we observe cases of drops detaching from the substrate after substantially shorter contact times than in Regime I, on the order of $10-20 \mathrm{~ms}$. The number of vapour structures substantially increases, while their average size and lifetime decreases (seen from TIR, Fig. 2(b) and particularly well in $\mathrm{X}$-ray, Fig. 3(b), $t=0.2-1.1 \mathrm{~ms}$ ). Often, the drop fragments in a spread-out state, particularly well seen in top view (see Fig. 10). Similar lift-off of drops after contact with a hot substrate was observed before 34 39, but never analyzed even qualitatively. Generally, drop bouncing from hot substrates was regarded to 
be due to the formation of a complete vapor layer between the drop and the substrate ${ }^{16}$, i.e. the dynamic Leidenfrost transition. However, our experiments evidence than the bare transformation of surface energy to kinetic energy upon drop retraction together with a sufficiently rapid receding contact region are enough to cause a drop to lift off the hot substrate, even if the Leidenfrost transition is by far not reached. As this small contact region is usually close to the impact point, we can clearly identify local, moving contact points separated by vapor bubbles in the X-ray data of Fig. 3(b), $t=9.2 \mathrm{~ms}$. We carried out at least 10 experiments per temperature, and we can observe both sticking and lift-off in individual impact events between $T_{s}=165^{\circ} \mathrm{C}$ and $168^{\circ} \mathrm{C}$, cf. also Sec. 4. At lower temperatures, only sticking is observed. This temperature range roughly coincides with the emergence of the transition boiling regime. Significant spray formation is characteristic for Regime II. The high-T range of Regime I and Regime II (Fig. 4(b)) correspond to the 'thermal atomization' observed and described in Ref. ${ }^{39}$.

Upon further increase of the substrate temperature, we enter Regime III: Contact is lost before the drop lifts off the substrate, as seen in Figs. 2(c), 3(c) and 4(c). The central region of contact becomes smaller and more porous. If spray is observed, it occurs sideways during the early spreading phase. Drops retract as a whole. In the example, $t_{l} \approx 26 \mathrm{~ms}$, while the TIR data reveal a more than ten times shorter contact time of only $t_{c}=1.6 \mathrm{~ms}$. The corresponding X-ray images, Fig. 3(c), are characterized by smooth, slowly evolving liquid-vapor interfaces after contact is lost (shown in the image for $t=13.8 \mathrm{~ms}$ at $T_{s}=182^{\circ} \mathrm{C}$ ). At high temperatures, approaching the dynamic Leidenfrost point, we observe a slight delay of a few microseconds between impact and initial contact in both TIR and X-ray data. We suppose that this can be related both to substrate cooling similar to the drops impacting on glass in Ref. $\frac{18}{}$ and to the presence of small surface or liquid contamination due to dust. In particular, there is a broad range of $\approx 40^{\circ} \mathrm{C}$ above the dynamic Leidenfrost point, where short, delayed contact can be observed in some realizations. Thus, for individual impact events, we may observe regime III and the Leidenfrost regime IV at the same substrate temperature.

In regime IV, the Leidenfrost or film boiling regime, the drop continuously levitates on a vapor layer and never contacts the substrate. In TIR, a spreading, grey-scale ring may be seen in particular at lower temperatures, where the vapor layer is sufficiently thin such that the evanescent wave is partially transmitted (see Ref. ${ }^{63}$ for details). In the X-ray data, the drop's bottom is perfectly smooth. As the drop spreads, a vapor rim forms behind the droplet's rim, and capillary waves evolve on the lamella during retraction 3132 . The drops rebound by contracting to a slender liquid column from which several smaller satellite drops are usually ejected upwards. In the example of Fig. 2(d), 6 satellites are formed starting after $t=13 \mathrm{~ms}$. Rebound occurs after $t_{l}=16.75 \mathrm{~ms}$. The maximum levitation of the drop's bottom is $\approx 4.4 \mathrm{~mm}$, reached after $44 \mathrm{~ms}$ (Determination of the height of the center of mass is complicated as the drop decomposes and parts of it leave the field of view.).

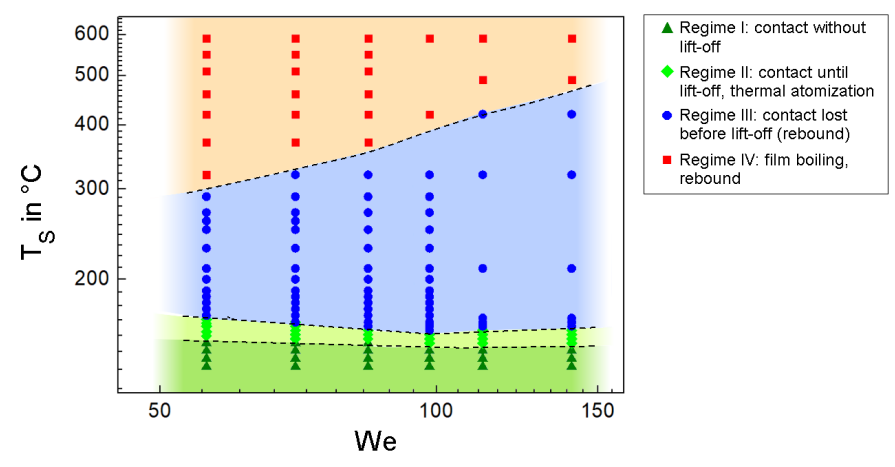

Fig. 5 State diagram for ethanol drop impact on a heated silicon substrate with different We showing the four different impact regimes (see text and Figs. 2 3 4). Dashed lines are guides to the eye indicating the Regime transitions.

Finally, we report the occurrence of the respective regimes at varied impact Weber number We extracted from X-ray experiments of $2 \mathrm{~mm}$ diameter ethanol drops impacting on a silicon substrate in the state diagram in Fig. 5. The points are deduced from 5 to 10 repeated experiments for each point. Only the dynamic Leidenfrost temperature (transition of regime III $\rightarrow$ IV) strongly increases with increasing impact velocity (Weber number), while the cross-over between the contact / lift-off regimes display no (I $\rightarrow$ II, at $150^{\circ} \mathrm{C}$ ) or little (regime II $\rightarrow$ III, between $160^{\circ} \mathrm{C}$ and $170^{\circ} \mathrm{C}$ ) temperature dependence within experimental accuracy. The irrelevance of the Weber number (at constant drop size) for the existence of regime II (similar to thermal atomization) is in accordance with the work of Roisman et al. 39 . This transition is mainly related to the evolving thermal and viscous boundary layers in the spreading drop.

\subsection{Contact and Lift-off Times on Silicon and Sapphire}

The lift-off time $t_{l}$ of an impacting drop refers to the moment when the entire drop (including all its visible fragments) retracts away from the substrate's surface at some velocity, leaving a clearly visible gap in side views. This corresponds to the usual 'contact' time determination in most of the literature, and its appearance is similar in optical and X-ray imaging (Here, in X-ray, the drop size exceeds the field of view.). In a large parameter range at high temperatures (Regimes III and IV), $t_{l}$ by far exceeds the actual contact duration $t_{c}$.

\subsubsection{Method and Validation:}

Determination of the contact time is obvious in TIR images far from $T_{L, d}$, see Fig. 22 It corresponds to the disappearance of all dark (wetted) spots. As TIR can only be applied to transparent substrates, we develop a new method to extract the contact time from our phase contrast X-ray data in order to access contact times on arbitrary smooth surfaces. So far, the literature investigating impacting drops using X-rays only used the easily visible absorption contrast between different fluids in transmis- 
(a)
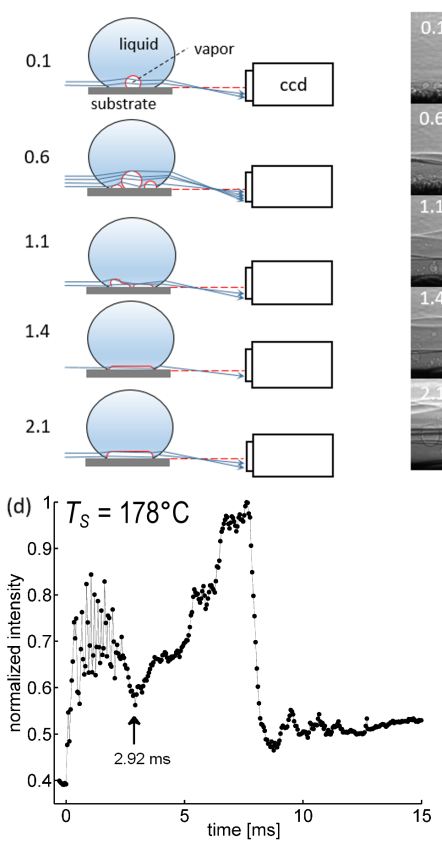

(f)

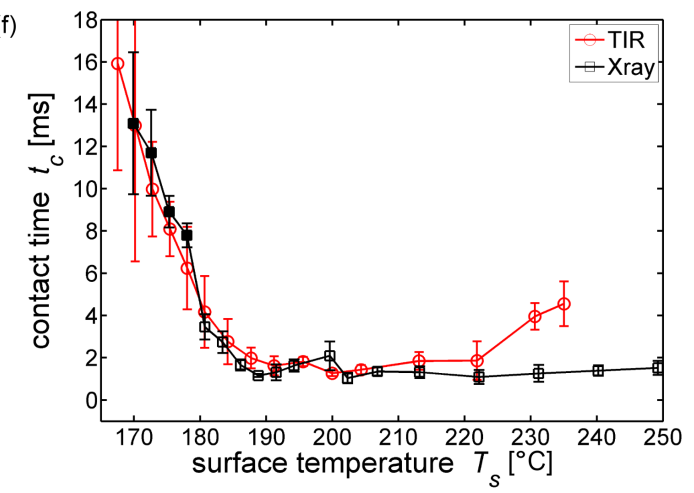

Fig. 6 Contact time determination from X-ray refraction: (a) schematic, $X$-ray enters parallel to the substrate's surface and gets partially refracted at corrugations of the phase boundary at the drop underside (blue arrow line). The cumulative refracted beam intensity increases with larger spreading radius of the drop and with larger and more numerous vapour structures, and it decreases as bubbles coalesce and the vapor film flattens. (b,c) Representative X-ray snapshots for (b) the growth and merging of vapor structures and (c) the refracted X-ray beam below the substrate in a small box from (b). (d,e) Exemplary normalized mean intensity evolution from a box of $1 \mathrm{~mm}$ width around the impact point, depth $6-46 \mu \mathrm{m}$ below the surface, (d) at the onset of Regime III for $T_{S}=178^{\circ} \mathrm{C}$ and (e) $T_{S}=200^{\circ} \mathrm{C}$. Arrows mark the local minima corresponding to $t_{c}$. (f) Comparison of the contact times on sapphire obtained from TIR and $\mathrm{X}$-ray refraction, averages over at least 10 individual experiments, error bars correspond to the standard deviation. X-ray data for $T_{S}<177^{\circ} \mathrm{C}$ were obtained from side view (filled symbols), for $T_{S}>177^{\circ} \mathrm{C}$ from the refraction (empty symbols). All data: ethanol on sapphire, $D_{D}=2 \mathrm{~mm}$, $U_{0}=1 \mathrm{~m} / \mathrm{s}$. sion and the slight refraction of the X-rays at the gas-liquid interfaces $29 / 31 / 32 / 64 \mid 66$, whose interpretation was outlined above and in Fig. 1(e). Apart from these obvious features, we observe an $\mathrm{X}$-ray refraction below the substrate's surface position in the images when the drop's surface is sufficiently close to the substrate. Fig. 6(a) shows a sketch of the geometry. The overall appearance of the refraction-and-absorption induced image near the impact location is shown in Fig. 6(b). Images in (c) show the small contrast-enhanced detail in a small box from (b), right below the substrate's top surface. We will exploit exactly this refraction to determine the contact time when it is not directly accessible in side view (mainly Regime III).

The mechanism is as follows: The X-rays penetrate through the drop and are partly reflected and partly refracted at the phase boundary between the liquid and the vapor underneath, which weakly visualizes the internal vapor structures. The refracted beam can reach below the substrate as shown by the blue arrows in Fig. 6(a). This increases the intensity below the substrate's surface locally, see (c,d,e).

For the extraction of the contact time, we only consider mean intensities in a predefined region: First, we determine the impact point and the substrate's top edge as reference point. Next, we sum the intensity for each frame in a fixed region of interest. Our standard choice was a box height of $40 \mu \mathrm{m}$ (20 pixel) starting downward at $\approx 6 \mu \mathrm{m}$ below the surface. The width of the box was $1 \mathrm{~mm}$ (500 pixel) centered around the impact point. The box size was accordingly smaller if this maximal distance exceeded the image size, which occurred due to off-centered impacts or due to limited frames dimensions at frame rates exceeding 20889 fps. Next, we determined the mean intensity in the box for each frame ${ }^{\dagger}$. Fig. 6 (d) and (e) show two exemplary intensity curves at different temperatures, normalized to their maxima.

In correspondence with our TIR results and the side view X-ray images, we interpret the data as follow $\$$. The intensity remains spatially and temporally uniform at some reference value while the drop approaches the substrate, see data for $t<0$. As soon as numerous small vapor bubbles and / or channels are formed due to liquid-substrate contact, the refraction displays spatially inhomogeneous, fluctuating behaviour above those regions where the contacts occur. The mean intensity increases and reaches a first broader maximum (e.g. around $2 \mathrm{~ms}$ in (d)). Those local changes in intensity are inevitably connected to the evolution of the boiling patterns underneath the drop, i.e. the intensity changes become smaller and slower with increasing average size of the bubbles, and the reflection image becomes rather smooth again over regions where the vapor layer has formed and the interface dynamics calm down. The oscillatory behaviour of the intensity before $t=3 \mathrm{~ms}$ in (d) is not an artifact, it corresponds to the oscillatory wetting dynamics 2526 .

After that, the intensity decreases to a clear local minimum.

$\dagger$ Note that the small cut-out in Fig.6(c) is only illustrative, it is much smaller than the actual box used for the contact time evaluation from mean intensities, e.g., shown in in $(\mathrm{d}, \mathrm{e})$.

$\ddagger$ For a slightly tilted substrate, the reflection seen in Fig. 1.(c) displays the same features. 
This is related to a reduction of the number and increase of the lateral length scale of the vapour structures, i.e. merging bubbles and growing vapour channels, which we likewise observed in TIR. In Fig.6(b), one also observes that the mean height of the small scale vapour structures decreases, compare the frames at $t=0.6 \mathrm{~ms}$ and $t=1.1 \mathrm{~ms}$. Even the large central bubble calms and decreases in height. As a last aspect, it is characteristic for the transition boiling regime that the contact radius reduces (seen in our TIR data, e.g. Fig. 11 and reported in Refs.17|18), finally producing a continuous vapor film underneath the entire drop. In this moment, the vapor film's thickness is assumed to be minimal, resulting in the local minimum of the refraction intensity marked by the arrows in Fig. 6(d) and (e) after $2.9 \mathrm{~ms}$ and $1.4 \mathrm{~ms}$, respectively. The intensity does usually not grow monotonously afterwards, as the vapour layer thickness is inhomogeneous due to the dynamics of the droplet (in contrast to a true Leidenfrost drop in Regime IV). Eventually, the intensity will decrease again until complete retraction of the drop from the heated substrate.

Consequently, we identify the contact time $t_{c}$ with the occurrence of the minimum in the local averaged refracted intensity in a large region around the location of initial impact. Contact times measured from X-ray and TIR, averaged over at least 10 individual experiments at each temperature, are in excellent agreement in Fig. 6(f). At high temperatures, there is a small delay between impact and the first contact, which is within the error bar. Moreover, contacts are extremely fast, sparse and can be re-entrant, i.e. dissappear completely during a transient period. In the intensity curves, the initial peak can become shallow, however, the local minimum is still visible. This causes the discrepancies between the data sets, and the increase of $t_{c, \text { TIR }}$ above $T_{S}=220^{\circ} \mathrm{C}$. X-ray data for $T_{S}=170^{\circ} \mathrm{C}$ are unavailable due to the limit of exposure of the scintillator.

Note that the exact selection of the region under the substrate's surface is not important for the contact time determination, in general: Most changes in the box parameters only affect the relative height of intensity minima and maxima, but not their moment of occurrence $t$. Changing the height and (within small range) depth of the box beneath the substrate only changes the intensity amplitude. The evaluated region must be wide enough to capture a representative contact region underneath the drop. As shown in Fig. 2(b,c), also Fig. 11(c), the longest persisting contacts appear near the impact point, here within approx. $1.5 \mathrm{~mm}$ distance from the location of impact for the analyzed parameters. In principle, one can average over the full frame width. For large image widths, this has the disadvantage of averaging the signal too much. Alternatively, one can analyze the intensity profiles from several smaller boxes at different locations under the drop, and take the latest occurrence of the minimum. Too small box sizes cause disadvantageous signal to noise ratios.

\subsubsection{Contact and Lift-off Times:}

The lift-off and contact times of ethanol drops impacting on sapphire and silicon substrates in dependence on the initial surface temperature are presented in Fig. 7) over a large range of temperatures, spanning the four contact and impact regimes described in the previous section. The observations on both substrates are very similar. The contact time $t_{c}$ shown in (a) displays a strong, continuous decrease between $T_{s}=160^{\circ} \mathrm{C}$ and $200^{\circ} \mathrm{C}$, and gradually decreases with further temperature increase. Note that this is accompanied by drastic changes of the morphology and area fraction of the global contact as well as duration of the local contacts, as described in Ref. ${ }^{25}$. Above $T_{s}=200^{\circ} \mathrm{C}$, contacts are very short-lived (lasting few microseconds) and localized to often sub-micrometer contact sites. In addition, the initial impact is contact-less, but recurring contacts appear with some initial delay, and possibly even intermediate contact-less periods appear at these high temperatures. This causes the increase in the measured contact times on sapphire (red curve in (a)) for $T_{s}>210^{\circ} \mathrm{C}$, as we measured the total duration between first and last visible contact. Thus, the determination of the contact time alone will be insufficient to characterize the heat transfer at high temperatures. Breitenbach et al. $\frac{34}{\text { reported temperature-independent }}$ contact times of water droplets with a smooth surface in the temperature range of $200 \ldots 290^{\circ} \mathrm{C}$, where 'thermal atomization' of the drops occurs, based on top view imaging. This corresponds to the high-temperature range of Regime I, and II in our measurements. They developed an empirical model, where basically the duration of contact corresponds to the moment when the thickness of the thermal boundary layer reaches the thickness of the flattened lamella 39 , so that

$$
t_{c}=\frac{0.6 D_{D}^{2}}{b^{2} \alpha_{l} \operatorname{Re}_{0} 0.8}\left(\frac{v}{v_{0}}\right)^{0.8},
$$

where the $\alpha_{l}=k_{l} /\left(\rho c_{p}\right)$ is the thermal diffusivity, $k$ is the thermal conductivity, $\rho$ the density and $c_{p}$ the isobaric specific heat capacity, and $v$ is the kinematic viscosity of the liquid at the specific superheat temperature. The Reynolds number $\operatorname{Re}_{0}$ and the kinematic viscosity $v_{0}$ are taken at room temperature. From data taken at different Weber numbers, Roisman et al. ${ }^{39}$ could deduce that the constant $b=1.0$ for water. The model's prediction agrees very well with the data given in Ref. $\frac{39}{39}$ for water. Comparing to our contact times of ethanol drops, Fig. 7(a), no range of (even roughly) constant $t_{c}$ for $T_{s}<200^{\circ} \mathrm{C}$ exists, in qualitative disagreement to Ref. 39 . One might at first assume that this may be due to changing material parameters with increasing temperature, but this is not the case: Inserting the temperature-dependent thermal diffusivity and kinematic viscosity of ethanol into Eqn. (1), based on the thermal conductivity $k$ calculation from Assael et al. 69 and the other temperature-dependent material parameters given in the DDBST Dortmund data base (e.g. Refs. ${ }^{53}$ 54 ), and using $b=1.0$, we obtain contact times $t_{c}$ between 7.5 and $7 \mathrm{~ms}$ with a decreasing trend in the range between $T_{s}=165^{\circ} \mathrm{C}$ and $200^{\circ} \mathrm{C}$. This order of magnitude is agreeable below $180^{\circ} \mathrm{C}$, and changing $b$ does not change the qualitative trend. Again, the method of contact time determination may play an additional role: Our measured optimal lift-off times of $t_{l, \min } \approx 8 \mathrm{~ms}$, Fig.77(c), agree well with Roisman's estimation providing the time of lamella rupture. After rupture, the lamella retraction is expected to occur analogue to the rupture of low-viscosity liquid films presented by Taylor $\frac{44}{4}$ and Culick $\frac{45}{4}$, see also Ref. $\frac{43}{\text {. }}$. 
(a)

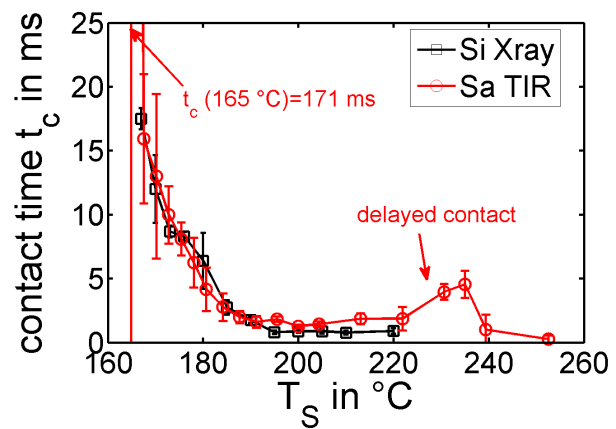

(c)

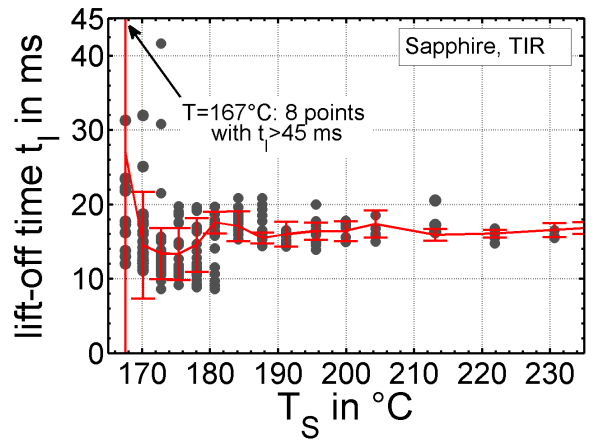

(b)

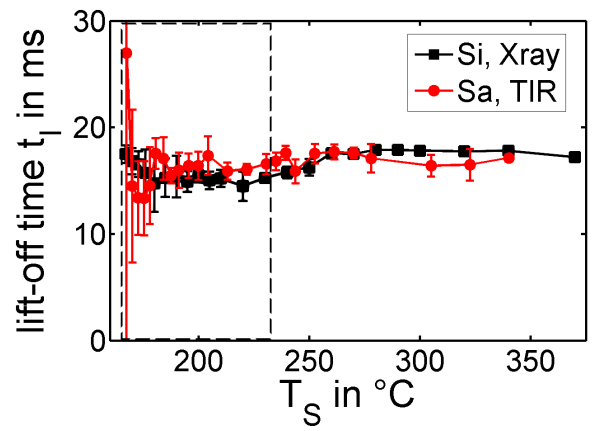

(d)

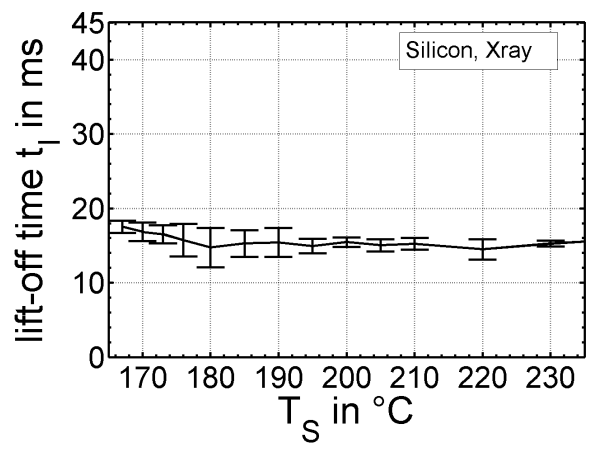

Fig. 7 Contact and lift-off times on bulk smooth sapphire (red circles, from TIR) and silicon (black squares, from X-ray) in dependence of the surface temperature at impact $T_{s}$ : (a) contact times, (b) lift-off times, (c) and (d) magnify the region in the dashed box in (b) for both substrates. The grey dots in (c) correspond to individual measurements, note that due to the large range of contact times below $T_{s}=175^{\circ} \mathrm{C}$, some data exceed the displayed range and are not shown. Error bars represent the standard deviation of each set of individual impacts under the same conditions. See Fig. 9 for more details. Points on the curve are average values, error bars indicate the standard deviation of that data set. Ethanol drops of $D_{D}=2 \mathrm{~mm}$ diameter impacting at velocity $1 \mathrm{~m} / \mathrm{s}, \mathrm{We}=71.2$.

Let us now analyze the duration that the drops reside in vicinity of the substrate, the lift-off times $t_{l}$, Fig. 7(b)-(d). At first glance, again the data on silicon and sapphire are similar and agree within the standard deviation of the data sets at given substrate temperature $T_{S}$. Contact and lift-off times in Regime I are on the order of few a seconds (not shown in the plot). Thus, the better thermal conductivity of silicon compared to sapphire has only small influence on the contact and lift-off times here. For impact on silicon (black squares, in (b) and (d)), we observe an initial slight decrease of the lift-off time below $T_{s}=180^{\circ} \mathrm{C}$, and an almost constant lift-off time for $180^{\circ} \mathrm{C} \leq T_{S} \leq 240^{\circ} \mathrm{C}$. Above $T_{s}=240^{\circ} \mathrm{C}, t_{l}$ slightly increases, followed by a slight decrease toward higher temperatures in the Leidenfrost Regime IV, continuing until $T_{s}=550^{\circ} \mathrm{C}$, where $t_{l} \approx 11.6 \mathrm{~ms}$. This slow decrease at high temperatures can be expected given that the vapor layer separating drop and substrate grows in thickness 29 . Thus, the small friction in the vapor layer decreases with increasing temperature, an one may presupposes that less surface energy is dissipated. Between $T_{s}=165^{\circ} \mathrm{C}$ and $240^{\circ} \mathrm{C}, t_{l, \mathrm{Si}}$ is roughly constant between $16 \mathrm{~ms}$ and $18 \mathrm{~ms}$. This experimentally determined rebound time agrees with the expectation from literature: There, it is approximated by the period of an oscillating drop 70 as $t_{l} \approx \pi / 4 \sqrt{\rho_{0} D_{D}^{3} / \gamma}=13.3 \mathrm{~ms}$, using the material properties at room temperature. Slightly larger empirical pre-factors than $\pi / 4$ were given by Biance et al. $\frac{51}{1}$ (prefactor $0.937, t_{l} \approx 15.8 \mathrm{~ms}$ ) and Chen et al. $\frac{52}{}$ (prefactor $1.12, t_{l} \approx 18.9 \mathrm{~ms}$ ).

The lift-off times on the sapphire substrates display a shallow local minimum between $T_{s}=170^{\circ} \mathrm{C}$ and $180^{\circ} \mathrm{C}$, where the mean lift-off time reduces to only $t_{l, \mathrm{Sa}} \approx 13.3 \mathrm{~ms}$, before it rises again for higher temperatures to $16 \mathrm{~ms}$ and $18 \mathrm{~ms}$ (the same value as measured on $\mathrm{Si}$ ). In the enlarged plot of Fig. 7(c), we added the values in the individual measurements. One observes that numerous points center around $\approx 10 \mathrm{~ms}$, whereas measurements with much higher lift-off times, occur at the same substrate temperature. More details and the mechanism behind this substantial lift-off time reduction are provided in Sec. 4.

Last, let us sketch the general time evolution of the contact characteristics of a drop impacting on a hot plate in terms of Fig. 8. Time evolves along the ordinate axis. The colored regions in the plots correspond to certain contact behavior: the blue region denotes contact with the substrate. In the red regions, the droplet is close to the substrate but contact-less (e.g. a Leidenfrost drop during spreading and receding), and the yellow region corresponds to times when the drop has accomplished its first liftoff or rebound. At low temperatures, the lift-off velocity is small and the re-approach of the droplet to the substrate occurs after very short time.

An ethanol drop impacting on a substrate at $T_{S}=150^{\circ} \mathrm{C}$ will remain in contact (blue region) all the time until it fully decomposed or evaporated (Regime I). At slightly higher temperature, $t_{c}=t_{l}$ (Regime II), i.e. the drop is initially attached to the substrate, but finally lifts off the substrate (direct transition from the blue to the yellow region). The width of this temperature range is only around $10 \mathrm{~K}$ (cf. also Fig. 5). For a broad range of temperatures, the contact is lost prior to lift-off or rebound (Regime 

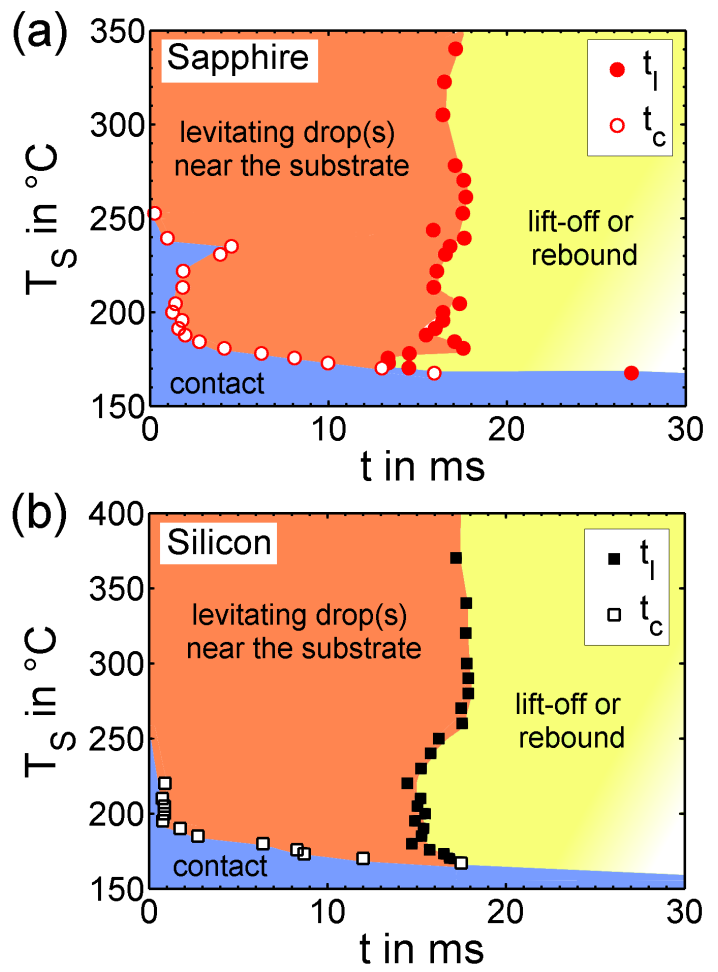

Fig. 8 Schematic evolution of boiling characteristics from impact until possible lift-off on (a) sapphire and (b) silicon based on the measured mean contact and lift-off times. Interpretation see text. Ethanol drops of $D_{D}=2 \mathrm{~mm}$ diameter impacting at velocity $1 \mathrm{~m} / \mathrm{s}, \mathrm{We}=71.2$.

III, range $170^{\circ} \mathrm{C} \leq T_{s} \leq 250^{\circ} \mathrm{C}$ on sapphire). This refers to a first cross-over from blue to orange, and then to the yellow region. Finally, in the Leidenfrost regime IV, drops spread and recede close to the substrate without any contact, before they rebound (transition red to yellow).

\section{Droplet Dynamics near the Minimum Lift-off Time}

In order to elucidate the origin of the lift-off time reduction on sapphire for surface temperatures between $T_{s}=170 \ldots 180^{\circ} \mathrm{C}$, we performed $\approx 25$ separate measurements for each temperature, and augmented the side and bottom TIR images with videos taken in a tilted top view perspective (see setup sketch in Fig. 1(b)). In this lift-off time reduction range, which makes up most of Regime II, droplets fragment into smaller drops, and lift off the substrate at comparatively low velocity in a flattened, disintegrated overall shape, see Figs. 2(b), 4(b). The transition from contact boiling (Regime I) to lift-off (Regime II) is not sharp. The two typical impact scenarios are sketched in Fig. 4(b):

In the lower temperature range of Regime II, we find individual impact events with long contact and lift-off times (tens to hundreds of milliseconds, not all data shown in Fig. 7) as well as such with very short ones (less than $10 \mathrm{~ms}$ ). In Figure9 we show the cumulative probability distributions of the lift-off times $t_{l}$ for different initial substrate temperatures $T_{s}$ : These distributions are usually non-Gaussian, often showing a population of impact events with short lift-off times and one with larger values.
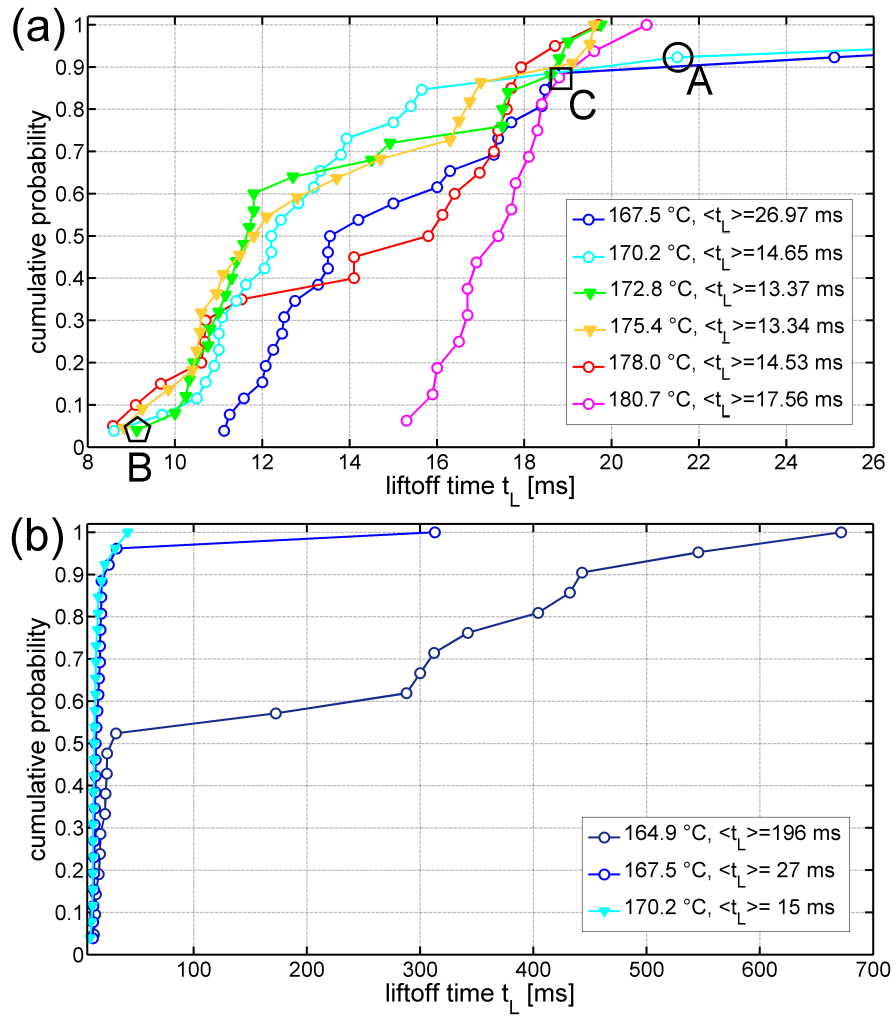

Fig. 9 Statistics of lift-off times in Regime II: Cumulative probability distributions of the lift-off time and mean lift-off times in dependence of the initial substrate temperature. The minimum occurs around $173 \ldots 175^{\circ} \mathrm{C}$ (green and orange curves, triangles), with average $t_{l, \min } \approx 13.3 \mathrm{~ms}$. The probability distributions below these temperatures possess 2 peaks, one at low, and one at high temperatures, seen by the drastic change of slope in the blue curves (b). Marked points in (a) correspond to image sequences in Fig. 10 Ethanol drops of $D_{D}=2 \mathrm{~mm}$ diameter impacting at velocity $1 \mathrm{~m} / \mathrm{s}$ on the sapphire substrate, measurements from side view imaging.

O (A) lamella rupture, but central drop sticks to substrate: $t_{\mathrm{C}}=20 \mathrm{~ms}, \mathrm{t}_{\mathrm{L}}=31 \mathrm{~ms}$

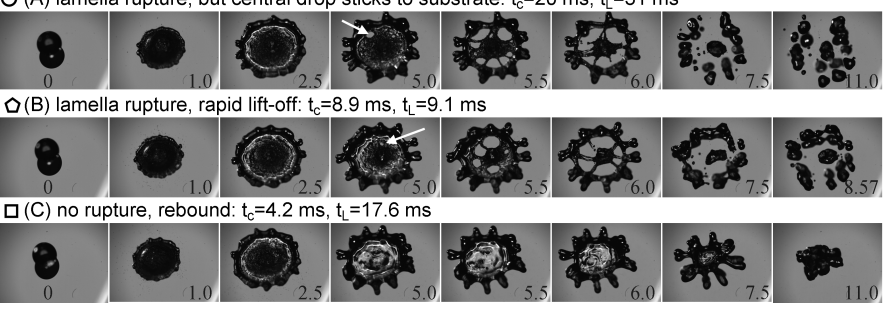

Fig. 10 Hole formation and sticking govern the lift-off, exemplary tilted top view image sequences corresponding to the marked points in Fig. 9(A): White arrows indicate early formed holes. (a) Lamella rupture with insufficiently fast retraction of the contact underneath the central drop region, central droplet is stuck, practically no lift-off. (B) optimal situations: hole expansion and sufficiently fast retraction of the contact (see also Fig. 11), lift-off in shape of a disintegrated pancake. (C) Increased temperature with rebound of a single drop, Regime III, heat transfer during spreading is insufficient to cause lamella rupture, $t_{c} \ll t_{l}$. The arrows in (A) and (B) indicate the first hole formed in the lamella. Numbers give the time after impact (identified in TIR) in milliseconds. Maximum spreading is reached after $\approx 6.35 \mathrm{~ms}$. Ethanol drops of $D_{D}=2 \mathrm{~mm}$ diameter impacting at velocity $1 \mathrm{~m} / \mathrm{s}$ on the sapphire substrate. 
(a)

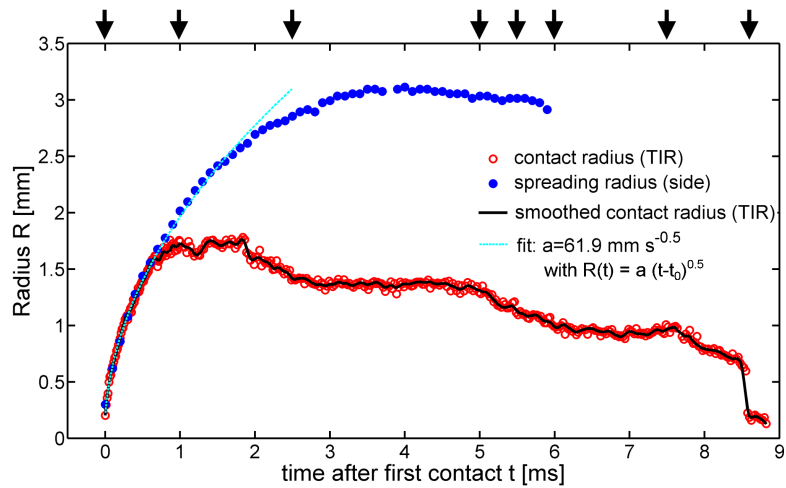

(b)

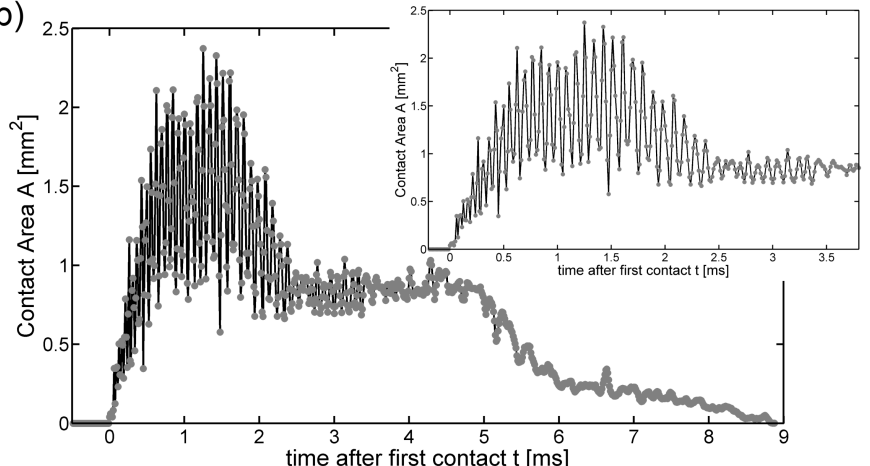

(c)

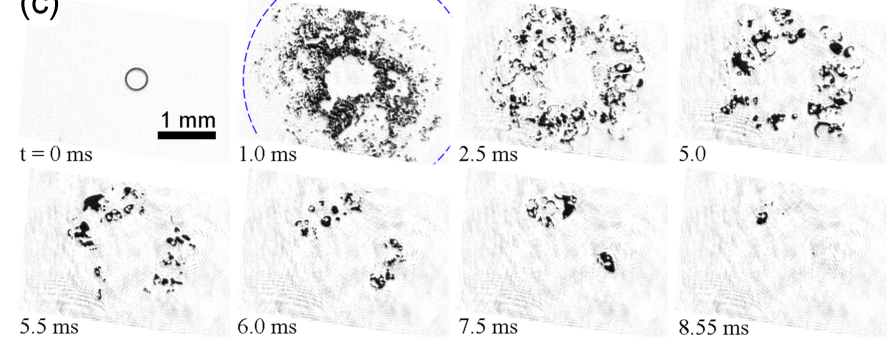

Fig. 11 Contact, hole formation and spreading dynamics for a drop with small lift-off time in the minimum region in Regime II, see Fig. 10(b): Ethanol drop of $D_{D}=2 \mathrm{~mm}, U_{0}=1 \mathrm{~m} / \mathrm{s}$, impacting on a sapphire substrate at initial temperature $T_{\mathrm{S}}=175.4^{\circ} \mathrm{C}$. (a) spreading radius, fit with $R(t)=a \sqrt{t-t_{0}}$, with $t_{0}$ the moment corresponding to zero contact radius, and $a=61.9 \mathrm{~mm} \mathrm{~s}^{-0.5}$. (b) Time dependence of the contact area measured by thresholding the TIR images: the contact and lift-off times approximately coincide, $t_{c}=8.9 \mathrm{~ms}$ and $t_{l}=9.1 \mathrm{~ms}$. (c) TIR images showing the contact morphology, corresponding to the times marked by arrows in (a), and to the tilted top view image sequence Fig. 10(B). The dashed circle at $t=1 \mathrm{~ms}$ indicates the contact radius in side view. At all later times, it by far exeeds the field of view of the TIR data.

The mean contact time continuously decreases in this range, cf. Fig. 7(a). For $T_{s} \leq 170.2^{\circ} \mathrm{C}$, we find an increasing number of impact events with $t_{l}<20 \mathrm{~ms}$, coexisting with impacts with very long contact and lift-off times of up to $0.7 \mathrm{~s}$, see Fig. 9 (b). At high temperatures, $T_{s}>180^{\circ} \mathrm{C}$, in Regime III, lift-off times display a narrow distribution between $15 \ldots 18 \mathrm{~ms}$. Drops rebound without disintegration, and this range coincides with expectations based on inertial, contact-less rebound. In the range of $170^{\circ} \mathrm{C}<T_{s}<180^{\circ} \mathrm{C}$, numerous impacts display a reduced lift-off time by up to a factor of $\approx 2$, to typically $t_{l}=7.8 \mathrm{~ms}$ and $11 \mathrm{~ms}$. The minimal mean lift-off time occurs around $T_{s}=(174 \pm 1)^{\circ} \mathrm{C}$, see green and orange curves in Fig. 9(a).
Tilted top view imaging indicates the mechanism behind this drastic contact time reduction, see Fig. 10 Lamella rupture causes droplet disintegration in a spread-out state, see (A) and (B). Such thermal atomization of a drop on a hot plate occurs when the temperature at the top of the spread-out lamella reaches the boiling point of the liquid 39 , and bubbles rupture through the liquid film. However, this alone is insufficient to cause the lift-off time reduction: Additionally, the lamella retraction must be easily possible, i.e. the contact between the substrate and the drop must recede sufficiently fast to avoid contact line pinning, as in (B). Only when both conditions are fulfilled, the lift-off time is substantially reduced.

Drop spreading / receding and contact dynamics in the situation of a short lift-off time are exemplarily analyzed in Fig. 11 corresponding to Fig. 10(B) and the Regime II in Fig. 4(b): The temporal evolution of the spreading radius (blue) and the radius of the smallest circle containing all contact spots (red) is shown in (a). For $t \geq 0.66 \mathrm{~ms}$, the contact radius is smaller than the spreading radius. The outer regions of the spreading lamella levitate, while the central regions remain in contact. This is characteristic for transition boiling of impacting drops ${ }^{17118}$. Drop spreading follows the $R(t) \propto \sqrt{t-t_{0}}$ scaling rigorously derived by Riboux and Gordillo 71 .

For $t>0.66 \mathrm{~ms}$, the contact radius first remains roughly constant and then slowly recedes. The contact area, see Fig. 11 (b), displays high-frequency oscillations for $t \leq 3 \mathrm{~ms}$. This phenomenon is described in Refs.25226. After that, the contact area decreases more rapidly. In particular, contacts group into several smaller spots instead of being equally distributed (cf. image sequence in (c)). This particular drop lifts off the substrate at $t_{l} \approx 9.1 \mathrm{~ms}$. Impact events with longer lift-off times at similar temperatures correspond to longer lasting contact of the central fragment of the drop, remaining stuck to the substrate after lamella rupture caused fragmentation, as e.g. for Fig. 10(A). It is always this central fragment of the initial drop which avoids the lift-off. Fragments resulting from breakup of the rim of the lamella levitate.

In the present experiments, the first holes in the lamella appear due to a growing vapor bubble while the drop is still spreading, indicated by arrows in Figs. 10(A) and (B). This process requires an efficient heat transfer from the substrate to the drop liquid, which only occurs while direct contact between the drop and the liquid persists, i.e. the levitating parts of the lamella can be assumed to heat up more slowly than the parts which are in contact with the substrate. This sets an upper limit to the regime of thermal atomization, which is caused by vapor bubbles rupturing through the lamella. Thus, when a large part of the lamella levitates already early during the spreading process, the lamella remains intact and the drop will rebound from the hot surface as a whole (see Fig. 10(C)).

Finally, we note that we have not observed a similar phenomenon for ethanol drop impact on silicon, where only X-ray data is available. Close inspection of the X-ray data did not indi- 
cate any lamella rupture, although otherwise the drop interaction with that substrate was very similar to the sapphire disks. More detailed studies would be needed to reveal the reason for this discrepancy, which could be related to heat transfer properties but also to changed wettability and thus contact line behavior of the vapor bubbles.

\section{Discussion and Conclusions}

We combine Ultrafast synchrotron phase contrast X-ray, highspeed Total Internal Reflection and conventional side and topview imaging to study the contact and rebound of ethanol drops impacting on smooth hot plates. Exploiting the weak reflection and refraction of X-rays on topographic structures on the underside of a boiling drop, we developed a new method of reliable contact detection applicable to droplets impacting on hot nontransparent substrates such as metallic plates. The method was validated by direct comparison of contact time measurements on sapphire between X-ray and (optical) Total Internal Reflection data, with excellent agreement. This finally allows us to avoid the ambiguities of typical contact time measurements from side and top view data, and it allows a direct quantitative comparison of the mean contact times on sapphire and the even better heat conductor silicon.

The contact times display a monotonous decrease with increasing temperature, and agree within error bars for both substrates except near the dynamic Leidenfrost point. At such high temperatures, contact usually occurs with a delay respective to the moment of impact. This may be interpreted as an effect of rapid substrate cooling due to the interaction with the colder, evaporating drop similar to van Limbeek's results regarding (badly heat conducting) glass substrates $\frac{18}{}$. Using our combined method, we identify localized, short-lasting contacts at substrate temperatures more than $50 \mathrm{~K}$ above the previously determined Leidenfrost transition (based solely on the initial impact and TIR intensity) 1726.

Drop lift-off from the heated substrate is observed also at substrate temperatures, where substantial contact with the substrate exists. To analyze this phenomenon more deeply, we measure the lift-off times of the droplets in side view. Impact events are classified into the four regimes sketched in Fig. 4, based on the occurrence and duration of contact and until lift-off. Between the classical contact and Leidenfrost impact regimes, we observe a large range of higher temperatures where droplets rebound from the hot substrate after initial contact. Here, the contact times are almost constant, slightly decreasing with increasing temperature, between $16 \mathrm{~ms}$ and $20 \mathrm{~ms}$ both on sapphire and silicon. The drops rebound in increasingly prolate shapes with increasing velocities for increasing substrate temperatures. A small range of $10 \mathrm{~K}$ to $15 \mathrm{~K}$ at low temperatures with approximately equal the contact and lift-off times of the drop exists. This regime coincides with the appearance of the thermal atomization regime 39 . However, the measured contact times qualitatively disagree with the model's predictions 39: The model predicts a constant contact time of $\approx 8 \mathrm{~ms}$, which is the correct order of magnitude, but the constancy of contact time is in sharp disagreement with our measurements. The discrepancy is not straightforwardly explained by a change of material parameters. Drops in this regime fragment into on the order of 10 smaller droplets, and lift off the substrate at small velocity in the shape of a disintegrated pancake. Only the transition between the short-lived contact and the Leidenfrost (film boiling) regimes is strongly temperature dependent.

Strikingly, on the sapphire substrate, the mean lift-off times of those droplets where contact and lift-off times are approximately equal is substantially smaller than at higher temperatures, even than that of Leidenfrost drops. The statistics of lift-off times in individual impact events in this temperature range display a nonGaussian distribution around the mean, with typically one subset of points at small lift-off times between 8 and $11 \mathrm{~ms}$ and a large range of other data for up to few hundreds of milliseconds. We identify an optimal timing of spontaneous lamella rupture caused by bursting vapor bubbles with the receding of the contact underneath the drop as the physical mechanism behind the contact time reduction by up to a factor of 2 (down to $\approx 8 \mathrm{~ms}$ ). At low temperatures, this regime is limited by persisting contacts underneath the spreading and receding lamella, causing strong contact line pinning after lamella rupture events. The upper temperature limit is set by the aspect that sufficiently large and lasting contacts are required for an efficient heat transfer. This is the precondition for the thermal boundary layer in the liquid reaching the top of the lamella, which sets the time of thermal atomization 39 . The transition boiling regime of drop impact on hot plates is characterized by the fact that an increasing outer part of the lamella levitates on vapor at earlier times with increasing plate temperature 17 . This reduces the heat transfer efficiency, such that lamella rupture is absent above a certain temperature; thus the rupturebased mechanism of contact time reduction is eliminated.

Similar contact time reduction and rebound of droplets in a spread-out (pancake) shape was reported on superhydrophobic substrates 4250 . Levitating drops over hot plates (with neglect of the vapor flow) are frequently seen as an ideal realization of super-hydrophobicity, as their advancing and receding contact angles equal $180^{\circ}$. Chantelot et al. $\frac{42}{4}$ studied water droplets impacting on a super-hydrophobic substrate with a topographic defect. By this method, they cause lamella rupture in a controlled position and time, and predict the contact time reduction using a simple model. For our drops impacting on a hot plate, lamella rupture is less controlled, and occurs at several positions at different times. The advancing contact angle is always effectively $180^{\circ}$ (except for the contact boiling regime), as the lamella levitates on a vapor layer. The resistance against receding of the drop upon lamella retraction is governed by the boiling-related receding of the drop's contact with the hot plate: The receding contact angle is large where the lamella levitates, but it is small (similar to the ethanol-sapphire contact angle) where contact persists.

Our study explicitly shows that the transparent sapphire substrates used in many optical experiments provide an equally good basis for the measurements of contact times as heated metal substrates. An exception may apply for water drops, due to their much larger latent heat of vaporization. We provided reliable data for the contact times, which significantly differ from 'contact' times in the literature, which are typically determined from conventional side and top view imaging. Contact 
times monotonously decrease with increasing temperature, and micrometer-sized localized contacts may last for only microseconds when approaching the dynamic Leidenfrost transition. Neither the absence of spray nor the disappearance of structures on the spreading lamella provide a reliable criterion for contact determination. Our data can form an initial step towards more detailed experimental studies and towards a reliable basis for developments of theoretical models of the coupled boiling-and impact dynamics of drops on hot plates. Details of the mechanism of drop fragmentation and rebound need to be analyzed. This mechanism can be potentially exploited as a route towards controlled droplet removal, e.g. by smart design of structured surfaces.

\section{Conflicts of interest}

There are no conflicts to declare.

\section{Acknowledgements}

The authors would like to acknowledge the staff at the 32-ID beamline at Argonne National Laboratory for their support in realizing the X-ray measurements. The use of the Advanced Photon Source, an Office of Science User Facility operated for the U.S. Department of Energy (DOE) Office of Science by the Argonne National Laboratory, was supported by the U.S. DOE under contract no. DE-AC02-06CH11357. S. H. L, M. K. and J. H. J. acknowledge support by the National Research Foundation of Korea (NRF) grant funded by the Korean government (NRF-2017R1E1A1A01075274) and Brain Korea 21 PLUS Project for the Center for Creative Industrial Materials. K. H. acknowledges the German Science Foundation (Deutsche Forschungsgemeinschaft, DFG) for funding within Grants HA-8467/1 and HA8467/2-1. D. L. acknowledges funding by ERC grant 740479DDD. K. H., M. R. and D. L. thank the Max Planck Center Twente for funding.

\section{Notes and references}

1 H. Boerhave, Lugduni Batavorum, Leiden, 1732, vol. 1.

2 J. G. Leidenfrost, De Aquae Communis Nonnulis Qualitatibus Tractatus, Ovenius, Duisburg, 1756.

3 C. Wares, Int. J. Heat Mass Transf., 1966, 9, 1153.

4 D. Quere, Annu. Rev. Fluid Mech., 2013, 45, 197-215.

5 D. Hall and I. Mudawar, Int. J. Heat Mass Transfer, 1995, 38, 1201-1216.

6 J. Kim, Int. J. Heat and Fluid Flow, 2007, 28, 753-767.

7 G. Liang and I. Mudawar, Int. J. Heat and Mass Transfer, 2017, 115, 1206-1222.

8 M. Panao and A. Moreira, Exp. Fluids, 2005, 39, 364-374.

9 K. S. Hamdan, D.-E. Kim and S.-K. Moon, Annals of Nuclear Energy, 2015, 80, 338-347.

10 A. Biance, C. Clanet and D. Quere, Phys. Fluids, 2003, 15, 1632.

11 J. H. Snoeijer, P. Brunet and J. Eggers, Phys. Rev. E, 2009, 79, 036307.

12 B. Sobac, A. Rednikov, S. Dorbolo and P. Colinet, Phys. Rev. E, 2014, 90, 053011.

13 J. C. Burton, A. L. Sharpe, R. C. A. van der Veen, A. Franco and S. R. Nagel, Phys. Rev. Lett., 2012, 109, 074301.

14 J. C. Burton, P. Y. Lu and S. R. Nagel, Phys. Rev. Lett., 2013, 111, 188001.

15 S. Waitukaitis, K. Harth and M. van Hecke, Phys. Rev. Lett., 2018, 121, 048001.

16 T. Tran, H. J. J. Staat, A. Prosperetti, C. Sun and D. Lohse, Phys. Rev. Lett., 2012, 108, 036101.

17 M. Shirota, M. A. J. van Limbeek, C. Sun, A. Prosperetti and D. Lohse, Phys. Rev. Lett., 2016, 064501.

18 M. A. J. van Limbeek, M. Shirota, P. Sleutel, C. Sun, A. Prosperetti and D. Lohse, Int. J. Heat and Mass Transfer, 2016, 97, 101-109.

19 J. M. Kolinski, S. M. Rubinstein, S. Mandre, M. P. Brenner, D. A. Weitz and L. Mahadevan, Phys. Rev. Lett., 2012, 108, 074503.

20 J. M. Kolinski, L. Mahadevan and S. M. Rubinstein, EPL, 2014, 108, 24001.

21 S. Mandre, M. Mani and M. P. Brenner, Phys. Rev. Lett., 2009, 102, 134502.

22 L. Duchemin and C. Josserand, Phys. Fluids, 2011, 23, 091701.

23 W. Bouwhuis, R. C. A. van der Veen, T. Tran, D. L. Keij, K. G. Winkels, I. R. Peters, D. van der Meer, C. Sun, J. H. Snoeijer and D. Lohse, Phys. Rev. Lett., 2012, 109, 264501.

24 M. Khavari, C. Sun, T. Tran and D. Lohse, Soft Matter, 2015, 11, 3298-3303.

25 K. Harth, M. A. J. van Limbeek, M. Rump, D. van der Meer, A. Prosperetti and D. Lohse, in preparation.

26 M. Khavari and T. Tran, Phys. Rev. E, 2017, 96, 043102.

27 P. Kant, R. B. J. Koldeweij, K. Harth, M. A. J. van Limbeek and D. Lohse, PNAS, 2020, 117, 2788-2794.

28 R. B. J. Koldeweij, M. A. J. van Limbeek, K. Harth, P. Kant, R. de Ruiter, H. Gelderbloom, J. H. Snoeijer and D. Lohse, submitted.

29 G. C. Lee, H. N. Ho, J. K. Tong, K. Kim, H. S. Park, K. Fezzaa and M. H. Kim, Int. J. Heat Mass Transf., 2018, 124, 11631171.

30 J. S. Lee, B. M. Weon, J. H. Je and K. Fezzaa, Phys. Rev. Lett., 2012, 109, 204501.

31 S.-H. Lee, S. J. Lee, J. S. Lee, K. Fezzaa and J. H. Je, Phys. Rev. Fluids, 2018, 3, 124308.

32 S. H. Lee, M. Rump, K. Harth, M. Kim, D. Lohse, K. Fezzaa and J. H. Je, submitted.

33 J. Bernardin, J. Clinton, A. Stebbins and I. Mudawar, Int. J. Heat Mass Transfer, 1997, 40, 247-267.

34 J. Breitenbach, I. V. Roisman and C. Tropea, Int. J. Heat and Mass Transfer, 2017, 110, 34-42.

35 V. Bertola, Int. J. Heat and Mass Transfer, 2015, 85, 430-437.

36 H. J. J. Staat, T. Tran, B. Geerdink, G. Riboux, C. Sun, J. M. Gordillo and D. Lohse, J. Fluid Mech., 2015, 779, R3.

37 G. Liang, S. Shen, Y. Guo and J. Zhang, Int. J. Heat and Mass Transfer, 2016, 100, 48-57.

38 C. E. Clavijo, J. Crockett and D. Maynes, Int. J. Heat Mass Transf., 2017, 108, 1714-1726. 
39 I. V. Roisman, J. Breitenbach and C. Tropea, J. Fluid Mech., 2018, 842, 87-101.

40 G. Cossali, M. Marengo and M. Santini, Exp. Therm. Fluid Sci., 2005, 29, 937-946.

41 A.-L. Biance, C. Pirat and C. Ybert, Phys. Fluids, 2011, 022104, 23.

42 P. Chantelot, A. M. Moqaddam, A. Gauthier, S. S. Chikatamarla, C. Clanet, I. V. Karlin and D. Quere, Soft Matter, 2018, 14, 2227-2233.

43 E. Villermaux, Annu. Rev. Fluid Mech., 2007, 39, 419-446.

44 G. I. Taylor, Proc. R. Soc. A, 1959, 253, 313-321.

45 F. E. C. Culick, J. Appl. Phys., 1959, 31, 1128-1129.

46 S. Kooij, R. Skis, M. M. Denn, E. Villermaux and D. Bonn, Phys. Rev. X, 2018, 8, 031019.

47 B. Neel and E. Villermaux, J. Fluid Mech., 2018, 838, 192221.

48 S. Poulain, E. Villermaux and L. Bourouiba, J. Fluid Mech., 2018, 851, 636-671.

49 Y. Wang and L. Bourouiba, J. Fluid Mech., 2018, 848, 946967.

50 Y. Liu, L. Moevius, X. Xu, T. Qian, J. M. Yeomans and Z. Wang, Nat. Phys., 2014, 10, 515-519.

51 A. Biance, F. Chevy, C. Clanet, G. Lagudeau and D. Quere, J. Fluid Mech., 2006, 554, 47-66.

52 R. H. Chen, S. L. Chiu and T. H. Lin, Exp. Therm. Fluid Sci., 2007, 32, 587-595.

53 DDBST GmbH, Dortmund Data Bank, http://ddbonline.ddbst.de/ VogelCalculation/VogelCalculationCGI.exe?component=Ethanol, 2019.

54 DDBST GmbH, Dortmund Data Bank, http://ddbonline.ddbst.de/ DIPPR105DensityCalculation/DIPPR105CalculationCGI.exe, 2019.

55 G. N. Muratov, Zh. Fiz. Khim., 1980, 54, 2088-2089.
56 D. V. Matyushov and R. Schmid, Int. J. Phys. Chem. Ber. Bunsen-Ges., 1994, 98, 1590-1595.

57 The Engineering Toolbox, https://www.engineeringtoolbox.com/ specific-heat-capacity-ethanol-Cp-Cv-isobaric-isochoric-ethylalcohol-d_2030.html, 2019.

58 S. J. Spencer, G. T. Andrews and C. G. Deacon, Semicond. Sci. \& Technol., 2013, 28, 055011.

59 H. R. Shanks, P. D. Maycock, P. H. Sidles and D. G. C, Phys. Rev., 1963, 130, 1743-1748.

60 Y. S. Touloukian (Ed.), in Thermophysical Properties of High Temperature Solid Materials, The Macmillan Company, New York, 1967, vol. 4, ch. 1, Section 1, pp. 8 - 47.

61 Crystan Ltd., Sapphire Data Sheet, 2019, https://www. crystran.co.uk/optical-materials/sapphire-al2o3.

62 D. A. Ditmars, S. Ishihara, S. S. Chang, G. Bernstein and E. D. West, J. Res. Ntnl. Bureau of Standards, 1982, 87, 159-163.

63 M. Shirota, M. A. J. van Limbeek, D. Lohse and C. Sun, Eur. Phys. J. E, 2017, 40, 54.

64 L. V. Zhang, J. Toole, K. Fezzaa and R. D. Deegan, J. Fluid Mech., 2011, 690, 5-15.

65 L. V. Zhang, J. Toole, K. Fezzaa and R. D. Deegan, J. Fluid Mech., 2012, 703, 402-413.

66 J. S. Lee, S. J. Park, J. H. Lee, B. M. Weon, K. Fezzaa and J. H. Je, Nature Commun., 2015, 6, 8187.

67 B. M. Weon, J. H. Je, Y. Hwu and G. Margaritondo, Phys. Rev. Lett., 2008, 100, 217403.

68 K. Harth, M. Rump, S. H. Lee, M. Kim, K. Fezzaa, J. H. Je and D. Lohse, in preparation.

69 M. J. Assael, E. A. Sykioti, M. L. Huber and R. A. Perkins, J. Phys. Chem. Ref. Data, 2013, 42, 023102.

70 K. Makino and I. Michiyoshi, Int. J. Heat Mass Transf., 1984, 27, 781-791.

71 G. Riboux and J. M. Gordillo, Phys. Rev. Lett., 2014, 113, 024506 . 